\title{
ABDOMINAL PAIN IN PREGNANCY
}

\author{
Geoffrey Chamberlain
}

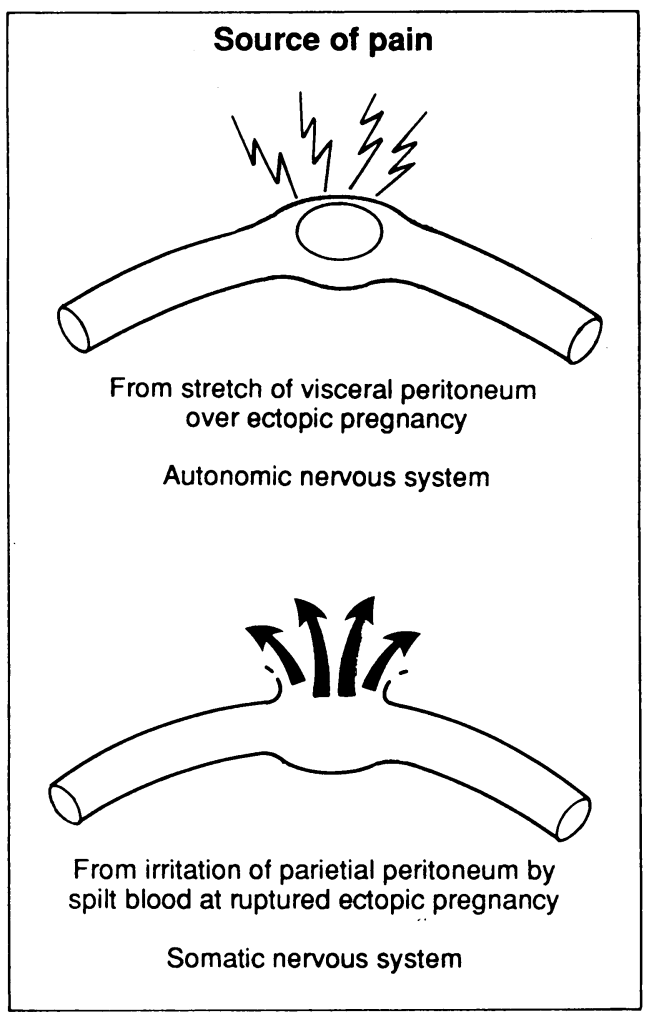

Pain in the stomach brings a woman to her doctor's surgery early, especially when she is pregnant. In most such cases the problem is not serious, but all cases need careful assessment as some women will need an immediate opinion at the hospital, some can wait till the next day, and others can wait till the next antenatal clinic. Abdominal pain is diagnosed mostly by clinical means. The history and examination are most important, investigations being less helpful than in other branches of medicine.

The use of ultrasonography by clinical gynaecologists has considerably improved the understanding of pelvic pathology. A vaginal transducer may give better images in early pregnancy than abdominal transducers; however, the laparoscope is still a major investigative tool for lower abdominal pain in early pregnancy. Under appropriate anaesthesia a good view of the pelvis and its organs clinches a diagnosis; in some cases treatment is also possible at the same time.

Pain arises either from inside an organ, involving the covering visceral peritoneum or from later involvement of the parietal peritoneum. The visceral aspects of the pain are poorly localised as they are mediated by the autonomic nervous system. Once the parietal peritoneum is affected, however, impulses travel by the somatic route and localisation may be more specific. Nausea and vomiting occur early in many pelvic conditions as well as in normal pregnancy, which might confuse the picture. Abdominal distension is not usual unless the alimentary tract is involved secondarily; furthermore, it is masked in mid and late pregnancy by the enlarging uterus. Shock may occur if acute pain is accompanied by sudden deterioration in the woman's condition such as in real hypovolaemia due to blood loss at a ruptured ectopic pregnancy or in relative hypovolaemia after excessive autonomic stimulation from peritoneal irritation of an abruptio placentae.

For the sake of analysis, in this article the commoner causes of abdominal pain in pregnancy are considered as occurring in either late or early pregnancy, but some conditions can occur in both. The causes will be considered under only one heading, which does not mean, however, that they do not occur in the other half of pregnancy.

\section{Early pregnancy}

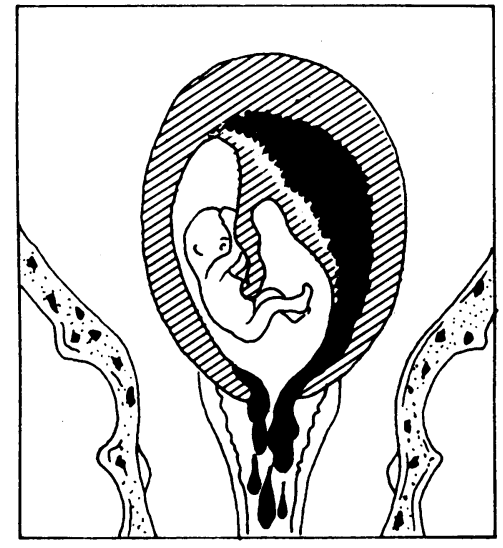

Inevitable miscarriage.

\section{From the uterus}

Abortion-One of the commonest causes of pain in early pregnancy is spontaneous miscarriage. The pain usually comes when the miscarriage has passed the threatened stage and is inevitable; the uterus squeezes clot and decidua through the cervical os, thus causing recurrent pains. The woman needs to be admitted to hospital for evacuation of the uterus. This subject is dealt with in more detail in the first of the articles on vaginal bleeding in early pregnancy. 

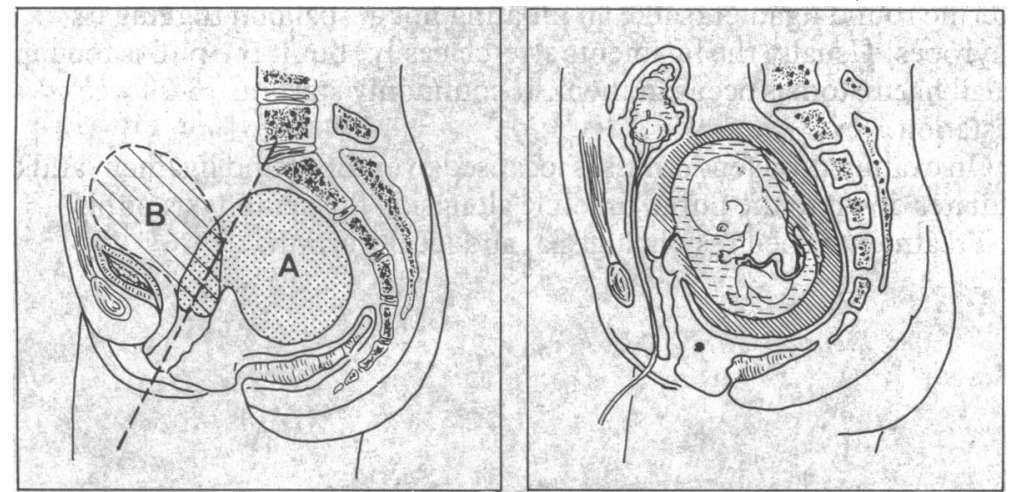

Left: Retroverted uterus (A) and anteverted uterus (B) in early pregnancy. Right: Management of impacted retroverted uterus during pregnancy (catheterisation).
Retroverted uterus - Retroversion is a common position for a normal uterus. In pregnancy the uterus expands into the abdomen. If adhesions are present, however, this cannot occur; by 10-12 weeks the enlarging uterus fills the pelvis and pain is associated with retention of urine. The urethra is stretched by the uterine bulk and the bladder pushed to the abdomen so that urine cannot pass. These findings can be confirmed by ultrasonography.

Management includes draining the urine with an indwelling catheter. There is little benefit from lying the woman on her front for a few days, although some doctors used to recommend this. The cure eventually comes when the uterus grows into the general abdominal cavity either by moving up entirely or by anterior sacculation, so relieving the urethral stretch.

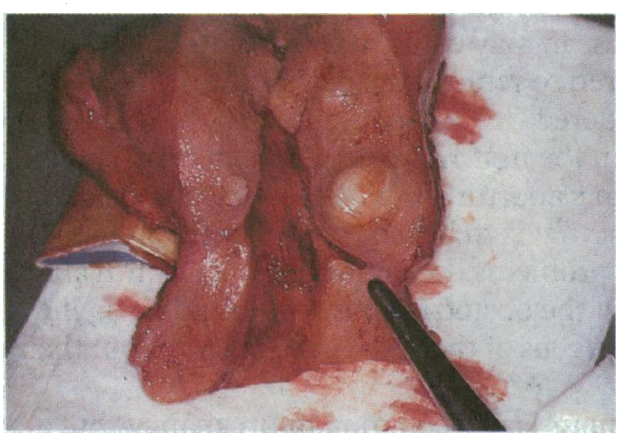

Fibroids are benign quiescent tumours consisting of whorls of fibres and a few cells.

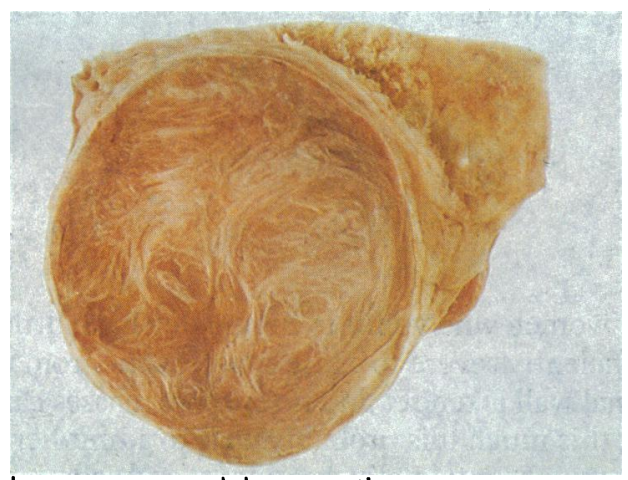

In pregnancy red degeneration may occur suddenly causing severe pain. The fibroid becomes much bigger and softer and is full of red cells. A transverse section looks similar to raw beef.
Fibroids-Fibroids are found in older pregnant women (those aged 30-40), particularly in West Indians. In pregnancy fibroids can undergo torsion if they are subserous; this is more common in the puerperium. Red degeneration is commonest at 12-18 weeks of pregnancy but can occur throughout, with resulting necrobiosis in the fibroid. The woman presents with tenderness over the mass accompanied by vomiting and mild fever.

Red degeneration is self limiting; if the diagnosis is firm, the best management is bedrest with analgesia and intravenous correction of the dehydration. Sometimes cases are doubtful, however, and ultrasonography may help to confirm the presence of fibroids, although necrobiosis may not show clearly. In truly doubtful cases, as in a low right sided fibroid that mimicks appendicitis, a laparotomy should be performed to exclude surgically correctable conditions. If red degeneration is then diagnosed the surgeon would do well not to remove the fibroid at this stage but to close the abdomen and continue conservative management.

\section{From the fallopian tube}

Ectopic pregnancy-An ectopic pregnancy is one of the most serious conditions that can occur in early pregnancy. Unruptured ectopic pregnancy causes chronic symptoms and needs to be managed in hospital whereas ruptured ectopic pregnancy produces acute symptoms and collapse and needs urgent hospital management. The condition is dealt with in detail in the second article on vaginal bleeding in early pregnancy.

Torsion-Torsion is uncommon and occurs mainly in younger women during early pregnancy when a long tube twists on its pedicle accompanied by torsion of the ovary. Torsion is due to the ovary and tube moving from a horizontal to a vertical position as the uterus enlarges.

The woman has non-specific hypogastric pain and a constant area of tenderness suprapubically on the lateral edge of the rectus abdominus muscle. Ultrasonography does not help but laparoscopy might be useful. A laparotomy is required; if the lateral end of the fallopian tube is nonviable it must be resected; occasionally the ovary is also ischaemic and requires removal. 


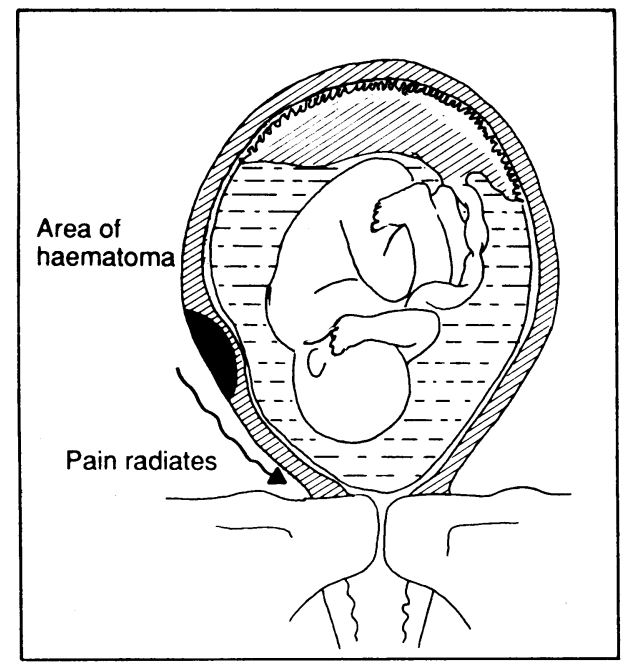

Haematoma of round ligament.

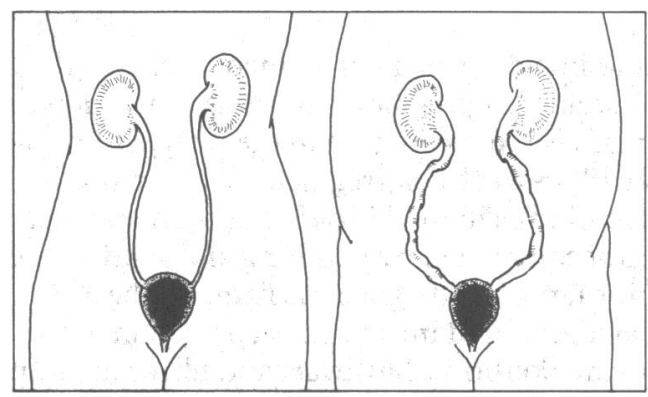

During pregnancy the ureters lengthen and become more tortuous and dilated.
From the pelvic ligaments

Round ligament-Stretch as the uterus rises in the abdomen pulls on the uterine round ligaments like an inflating hot air balloon tugging its guyropes. Usually the ligaments stretch easily, but if the pull is too rapid small haematomas occur in them. It commonly starts at 16-20 weeks' gestation.

On examination tenderness is localised over the round ligament and often radiates down to the pubic tubercle alongside the symphysis pubis.

Treatment is bed rest, analgesia, and local warmth.

\section{From the ovary}

Ovarian tumours - In early pregnancy an ovarian cystic tumour may rupture; bleeding may occur into a corpus luteal cyst, producing sudden pain localised poorly to one side of the abdomen with tenderness detectable on that side. An ultrasound scan may confirm the diagnosis, and a laparotomy is usually required to remove the part of the ovary containing the cyst. It used to be considered that a corpus luteum, the main site of progesterone manufacture in the first 12 weeks of pregnancy, could not be removed without the woman's aborting. This is no longer considered immutable. The trophoblast that is to become the placenta is already making progesterone; such problems do not usually occur until after the eighth week of pregnancy. If the obstetrician is concerned he or she could prescribe exogenous progestogens of the non-virilising type, but their use is unproved.

Torsion of an ovarian tumour is less common in pregnancy, but a dermoid may undergo twisting, producing colicky pain. A tender mass may sometimes be palpated, either abdominally or bimanually, and the treatment consists of removal of the tumour at laparotomy. This is safest between 14 and 24 weeks of gestation.

\section{Extrapelvic causes}

Vomiting - Though many women who vomit in pregnancy have little upset, vomiting may be sufficiently severe to cause muscle ache from the stretch. The upper abdominal wall is tender and no specific masses can be felt. If a woman is vomiting this much it is probably wise to admit her to hospital for intravenous treatment, antiemetic treatment, and sedation to allow her intestinal tract some peace. The pain usually settles down as the vomiting decreases.

Pyelonephritis - Stasis in the urinary tract associated with ascending urinary infection often follows dilatation of the ureters (due to raised progesterone concentrations) and the pressure of the increasing uterus on the bladder. It is most likely in early pregnancy, when the woman presents with vomiting, symptoms of fever, and low hypogastric or loin pain. She

- will be feverish and have ill defined tenderness over the suprapubic region and fairly precise tenderness in one or other subcostal angle. A midstream urine specimen may contain pus cells and bacteria.

Until the results of urine analysis are known the woman should have bed rest and be treated with local heat, a high fluid diet, analgesics, and broad spectrum antibiotics. The result of the urine test may indicate another antibiotic but often the patient's condition has improved by this time. Most women with pyelonephritis should be treated in hospital as intravenous fluid and antibiotics may be needed and labour may be stimulated by the accompanying fever. Recurrent or resistant urinary infection in pregnancy deserves follow up after the baby is born. A high proportion of such women have a structural abnormality of the urinary tract. 


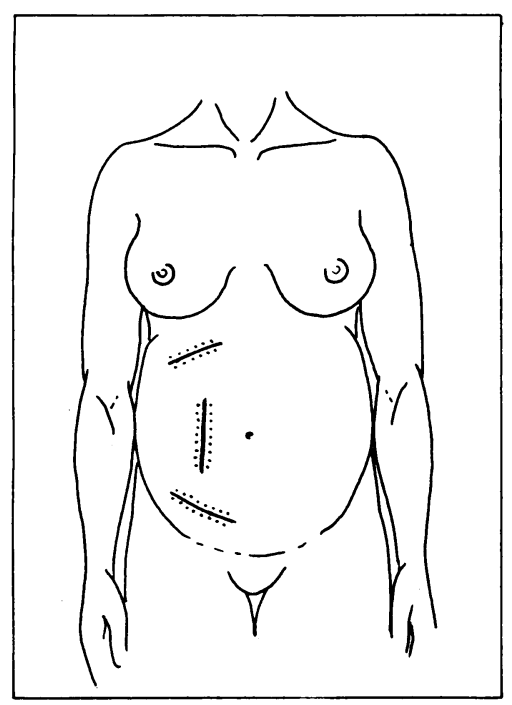

The site of incision for appendicectomy during pregnancy.

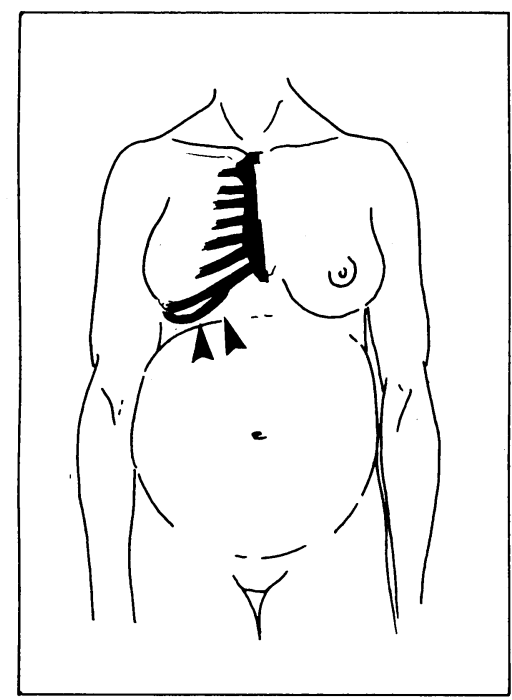

Pain in cholecystitis.

\section{Late pregnancy}
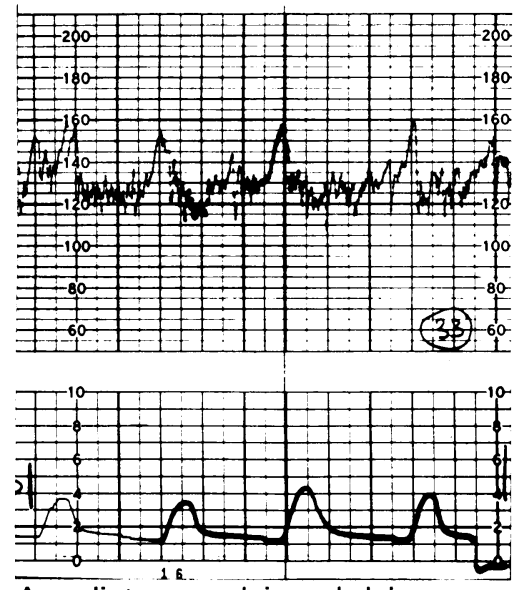

A cardiotocograph in early labour showing the fetal heart rate above the regular uterine contractions every three minutes.
Appendicitis - Appendicitis and pregnancy both occur in young women and therefore may occur concurrently by chance. The incidence of appendicitis in pregnancy is not increased but its diagnosis may be more difficult. For this reason and because of a reluctance to operate appendicitis used to have high rates of death and morbidity in pregnancy.

As it grows the uterus displaces the caecum from the right iliac fossa upwards and sideways, so the inflamed appendix may present with symptoms and signs in unexpected places. No longer tucked into the right iliac fossa, the appendix is present in the general abdomen and is less easy to wall off by omentum and gut when it becomes inflamed; generalised peritonitis is commoner in pregnant than non-pregnant women.

A history may elicit the characteristic pain shift, although it might not be localised to the right iliac fossa. Nausea and anorexia occur, sometimes confused by the symptoms of pregnancy. The tenderness over the appendix will shift higher as pregnancy continues. The treatment is operation, the incision being placed over the point of maximum tenderness marked by the surgeon before anaesthesia. Occasionally the results of a rectal examination can be falsely reassuring if the appendix has migrated from the area reached by an examining finger.

The previous reluctance to operate must be overcome; anyone suspected of having appendicitis in pregnancy should have a laparotomy by an experienced obstetric surgeon. Even in late pregnancy, caesarean section is not necessary at the same time unless the woman is in labour; women can have normal vaginal deliveries within a few days of an appendicectomy.

Other causes-Cholecystitis is commoner among women who live in or originate from countries whose residents characteristically have high cholesterol diets such as Australia and New Zealand. The pain is usually upper abdominal with tenderness centred on the eighth or ninth rib tip. Treatment in the absence of jaundice is drainage or removal, depending on the surgical need.

Volvulus of large bowel can occur in pregnancy, though it is characteristically more likely in the puerperium.

Small bowel colic may follow an attack of gastroenteritis. Urinary lithiasis occurs in the same frequency in pregnancy as outside pregnancy in areas of the country where the prevalence is increased.

\section{From the uterus}

Uterine contractions - All pregnancies end in labour, which can occur well before 40 weeks of gestation. Premature labour can present with abdominal pain, taking the woman and sometimes her general practitioner by surprise. Usually the pain is recurrent and the uterus can be felt contracting coincidentally with the pain. There may be a loss of mucus or a little blood from the vagina, and on vaginal examination the cervix is soft, thin, taken up, and sometimes dilated. When labour is very preterm (26-32 weeks) the woman should perhaps be transferred to a hospital with a good neonatal unit rather than to the one where she has booked (see the article on preterm labour).

Abruptio placentae-Separation of the placenta from its bed before the third stage of labour is painful and results in shock (see first article on vaginal bleeding in early pregnancy, $\mathrm{p}$ 1141). The pain is produced by blood tearing into the myometrium, separating the fibres widely, and finally reaching the visceral perineum. This also produces a tonic uterine contraction. Treatment is immediate delivery if the baby is still alive. 


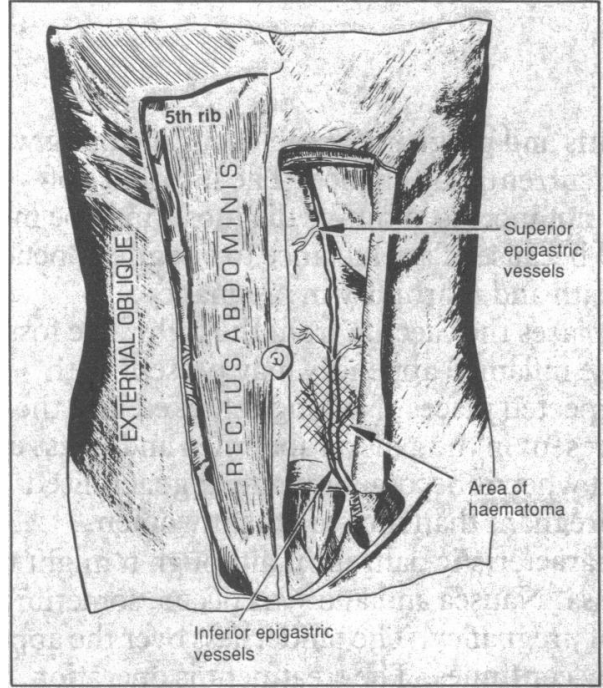

A rectus haematoma usually arises from the inferior epigastric vessels deep in the rectus muscle.

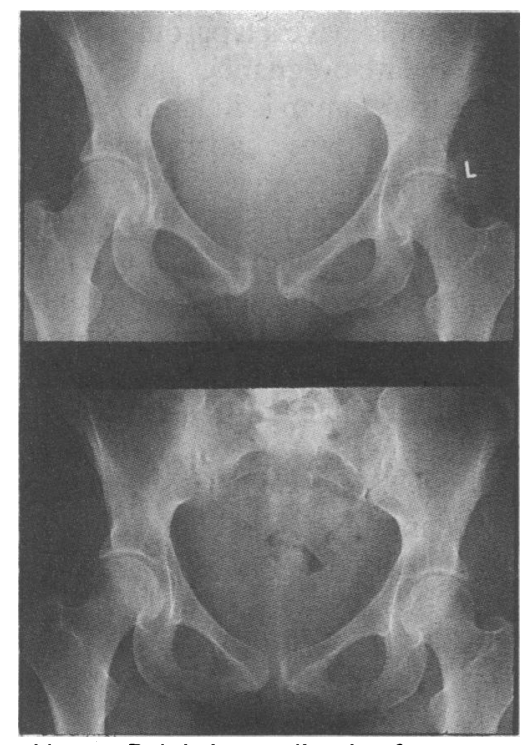

Above: Pelvis immediately after delivery showing dehiscence of pubis symphysis. Below: Same pelvis six weeks later.

\section{Conclusions}

Early abdominal examination will often help differentiate serious from non-serious conditions

\section{Extraperitoneal causes}

Pregnancy induced hypertension - In severe fulminating aspects of pregnancy induced by hypertension a woman may complain of hypogastric pain associated with vomiting. She will probably have raised blood pressure and proteinuria with oedema and be known to be hypertensive. There may also be visual signs (outlined in a future article on raised blood pressure in pregnancy). The pain is due to stretch of the peritoneum over the liver after the liver has become oedematous. Treatment of the pregnancy induced hypertension will resolve symptoms.

Rectus haematoma - Very rarely the rectus muscle may dehisce or the inferior epigastric veins behind the muscle rupture. As the anterior abdominal wall is greatly overstretched by the uterus, a fit of sneezing could cause this. Pain is severe and usually localised to one segment of the muscle. Blood loss is slight with the haematoma but increases if the veins rupture; investigations are not much help. Rectus haematoma is diagnosed from the fact that pain and tenderness persist when the woman contracts the rectus muscles by raising her head. Ultrasonography is helpful.

If the diagnosis is firm management is conservative, but in doubtful cases a laparotomy should be performed, and haematoma behind the rectus muscle confirms the diagnosis.

Pelvic arthropathy - Relaxation of the ligaments guarding the pelvic joints follows the secretion of the hormone relaxin. This allows appreciable separation of the symphysis pubis, giving abdominal pain that is much aggravated by walking. Treatment is rest; binders are of little help. It may take up to two months to resolve after delivery, but it usually does resolve.

Most women who present with abdominal pain in pregnancy may have nothing serious the matter. Pain can, however, lead the doctor to diagnose a serious condition, when action needs to be taken. As investigations play a small part in many of these diagnoses, experienced general practitioners can often diagnose cases and continue the management of many of them at home, but if there is any doubt the local obstetric department ought to be consulted.

Professor Geoffrey Chamberlain, FRCOG, is chairman of the department of obstetrics and gynaecology at St George's Hospital Medical School, London. 